\title{
A case of sigmoid endometriosis difficult to differentiate from colon
}

\section{cancer}

\author{
Philippos Dimoulios ${ }^{1}$, Ioannis E Koutroubakis*1, Maria Tzardi², \\ Pavlos Antoniou ${ }^{1}$, Ioannis M Matalliotakis ${ }^{3}$ and Elias A Kouroumalis ${ }^{1}$
}

\author{
Address: ${ }^{1}$ Department of Gastroenterology, University Hospital Heraklion, Crete, Greece, ${ }^{2}$ Department of Pathology, University Hospital \\ Heraklion, Crete, Greece and ${ }^{3}$ Department of Obstetrics and Gynecology, University Hospital Heraklion, Crete, Greece \\ Email: Philippos Dimoulios - fdimoul@hotmail.com; Ioannis E Koutroubakis* - ktjohn@her.forthnet.gr; \\ Maria Tzardi - tzardi_maria@yahoo.uk; Pavlos Antoniou - pantoniou_gr@yahoo.com; Ioannis M Matalliotakis - matakgr@yahoo.com; \\ Elias A Kouroumalis - kouroum@med.uoc.gr \\ * Corresponding author
}

Published: 07 August 2003

BMC Gastroenterology 2003, 3:18
Received: 15 May 2003

Accepted: 07 August 2003

This article is available from: http://www.biomedcentral.com/l47I-230X/3/I8

(C) 2003 Dimoulios et al; licensee BioMed Central Ltd. This is an Open Access article: verbatim copying and redistribution of this article are permitted in all media for any purpose, provided this notice is preserved along with the article's original URL.

\begin{abstract}
Background: Although endometriosis with sigmoid serosal involvement is not uncommon in women of childbearing age, the mucosal involvement is rare and differential diagnosis from colon cancer may be difficult due to the lack of pathognomonic symptoms and the poor diagnostic yield of colonoscopy and colonic biopsies.
\end{abstract}

Case presentation: We present a case of a young woman with sigmoid endometriosis, in which the initial diagnostic workup suggested colon cancer. Histologic evidence, obtained from a second colonoscopy, along with pelvic ultrasound findings led to the final diagnosis of intestinal endometriosis which was confirmed by laparoscopy.

Conclusion: Colonic endometriosis is often a diagnostic challenge and should be considered in young women with symptoms from the lower gastrointestinal tract.

\section{Background}

Extrapelvic endometriosis refers to endometriotic implants located elsewhere in the body including the gastrointestinal tract, urinary system, pulmonary system, CNS, skin and striated muscle $[1,2]$. The exact prevalence of extrapelvic endometriosis is unknown. The median age at the time of diagnosis is 34 to 40 years. Periodic hematochezia during the menses is a sign usually associated with intestinal endometriosis [3]. Yet, this catamenial character of bleeding and all other accompanying symptoms, is very often absent, making the clinical history rather misleading [4]. Moreover, the differential diagnosis of colonic endometriosis from other diseases of the colon is rather difficult due to the lack of pathognomonic symptoms and the poor diagnostic yield of colonoscopy and colonic biopsies.

In this report we present the case of a young woman with intestinal endometriosis, in which the initial diagnostic workup suggested colon cancer.

\section{Case report}

A 35-year-old woman was admitted to the Department of Gastroenterology because of rectal bleeding and bouts of 


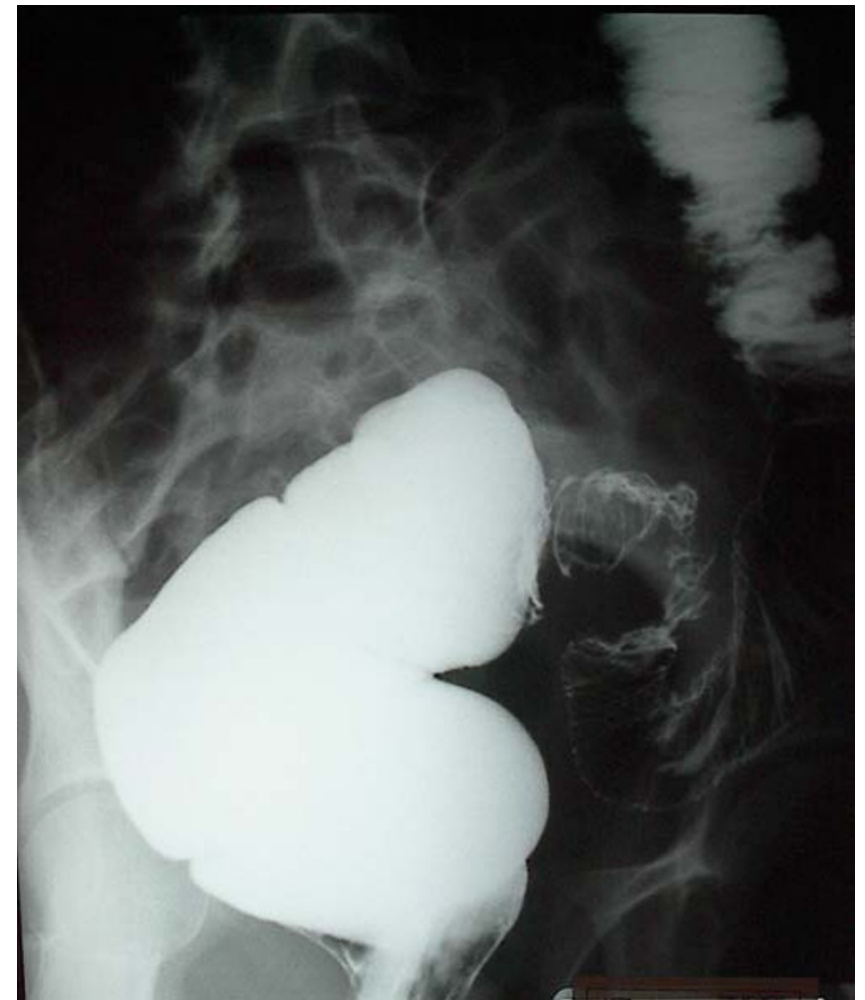

Figure I

Barium enema showing stenosis of the rectosigmoid region due to both a filling defect and an extrinsic bowel compression.

abdominal pain. Nine months before admission the patient began to have rectal bleeding that was related, at first, to the menstrual cycles. She also reported episodes of severe lower abdominal pain that was irrelevant to her menses and were accompanied by abdominal distention and constipation, especially during the last two months. Three weeks before admission she started to have almost daily hematochezia and small-caliber stools.

The patient had her menarche at the age of 13 years. Thereafter she had 27-day to 28-day menstrual cycles and menstrual periods lasting 6 to 7 days with normal blood loss. She had two normal labors at the age of 22 and 25. From her past medical history she reported tonsillectomy at the age of 11 and appendicectomy at the age of 18. She also reported cramping lower abdominal pain accompanying menstruation during the last three years. Her father suffered from hypertension. Her mother was healthy without reporting previous history of endometriosis or myomata uteri. Her brother was also healthy.

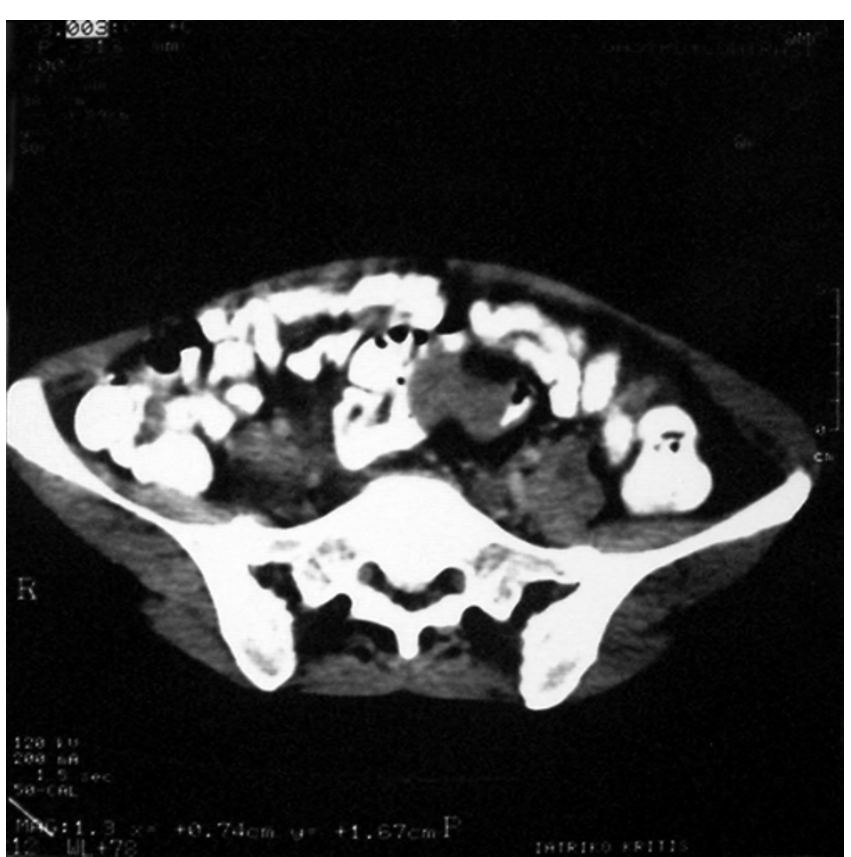

Figure 2

Eccentric wall thickening of the sigmoid with filling defect. Note also the ovarian cysts

Physical examination revealed mild lower abdominal tenderness. No masses were palpated. The bowel sounds were slightly increased. Rectal examination showed bright red blood, but no distinct mass. On the gynecological examination her vulva, vagina and cervix appeared to be normal. Her uterus had normal size and was anteverted. The laboratory work-up revealed a mild anemia (Hb: 11.3 gr/dL, Hct: 34.5\%, MCV: $91.7 \mathrm{fL}$ ) with an increased white

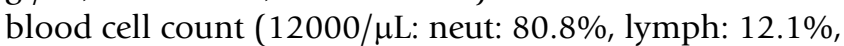
mono: $4.6 \%$ ) and ESR: $36 \mathrm{~mm}$. Coagulation parameters, serum urea, creatinine, electrolytes, and liver function tests, were all within normal range.

Colonoscopy revealed an extensive polypoid lesion of the mucosa, on the rectosigmoid junction, along with erythema, oedema, and ulcerations that resulted in stenosis of the lumen. The endoscope could not be introduced beyond the lesion. The histology of the above lesion demonstrated a mild dilation of crypts without goblet cell depletion. The lamina propria was edematous with dilated capillaries and inflammatory infiltrates of lymphocytes and plasma cells. A barium enema (figure 1), on the following day, showed stenosis of the rectosigmoid region due to both a filling defect and an extrinsic bowel compression. The rest of the colon was normal. An abdominal CT scan, obtained two days later, revealed an eccentric wall thickening of the sigmoid and confirmed 


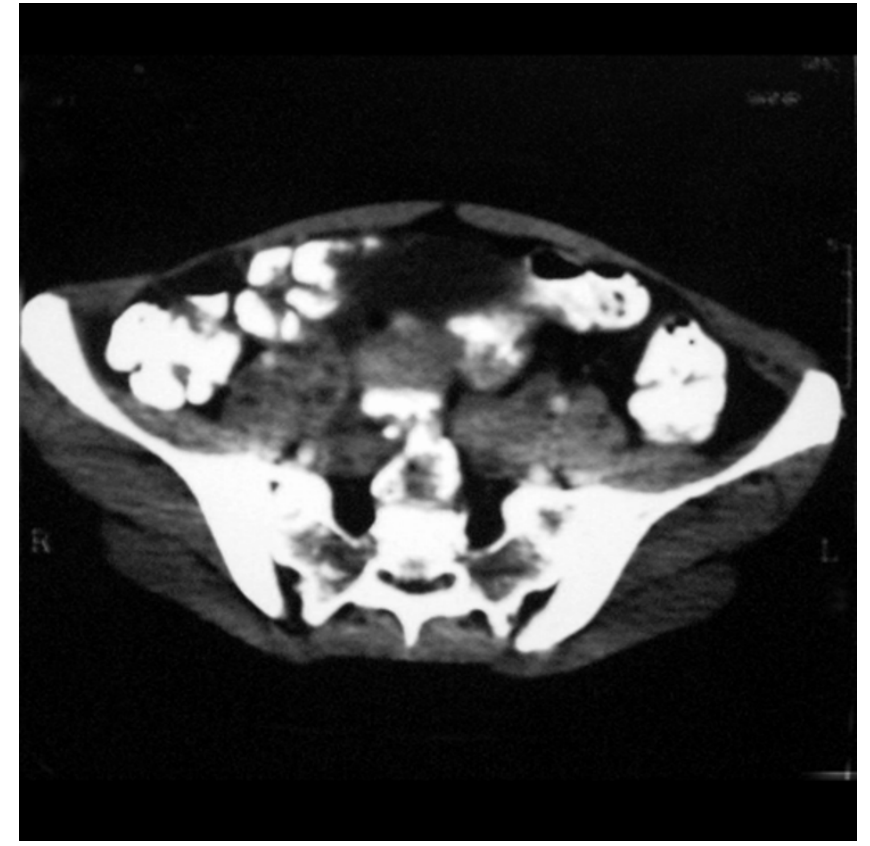

Figure 3

Eccentric wall thickening of the sigmoid with filling defect. Note also the ovarian cysts

the filling defect. Moreover, it showed ovarian cysts (Figures 2,3).

Because of high suspicion of colon cancer a new colonoscopy was performed, in the $20^{\text {th }}$ day of the menstrual cycle, in order to obtain more biopsy specimens. This time, colonic histology showed stroma and glands of endometrium suggestive of endometriosis (Figure 4).

Serum level of CA-125, that was measured in the $5^{\text {th }}$ day of her menstrual cycle, was $210 \mathrm{IU} / \mathrm{ml}$ (normal< $35 \mathrm{IU}$ / $\mathrm{ml}$ ), while CEA was $1.7 \mathrm{ng} / \mathrm{ml}($ normal $<5)$ and CA 19-9 was $6.1 \mathrm{IU} / \mathrm{ml}$ (normal<37). A pelvic ultrasound was performed and showed an anteverted homogeneous uterous. The right ovary had normal size with a small chocolate cyst $(3 \times 3.5 \mathrm{~cm})$, while the left ovary had also normal size, but a larger chocolate cyst $(4 \times 4 \mathrm{~cm})$. A laparoscopy performed a week later revealed stage IV endometriosis (American Fertility Society classification). The uterus was anteverted. Both the ovaries and the fallopian tubes were involved. The right ovary was found with a small chocolate cyst and the left ovary with a larger chocolate cyst (4 $\times 5 \mathrm{~cm}$ ). The cul-de-sac had extensive adhesions. An endometriotic implant was found on the sigmoid colon. All endometriotic lesions and adhesions were removed. The patient started treatment with a GnRH agonist (leuproreline acetate, $3.75 \mathrm{mg} / \mathrm{month} \mathrm{IM}$ ).

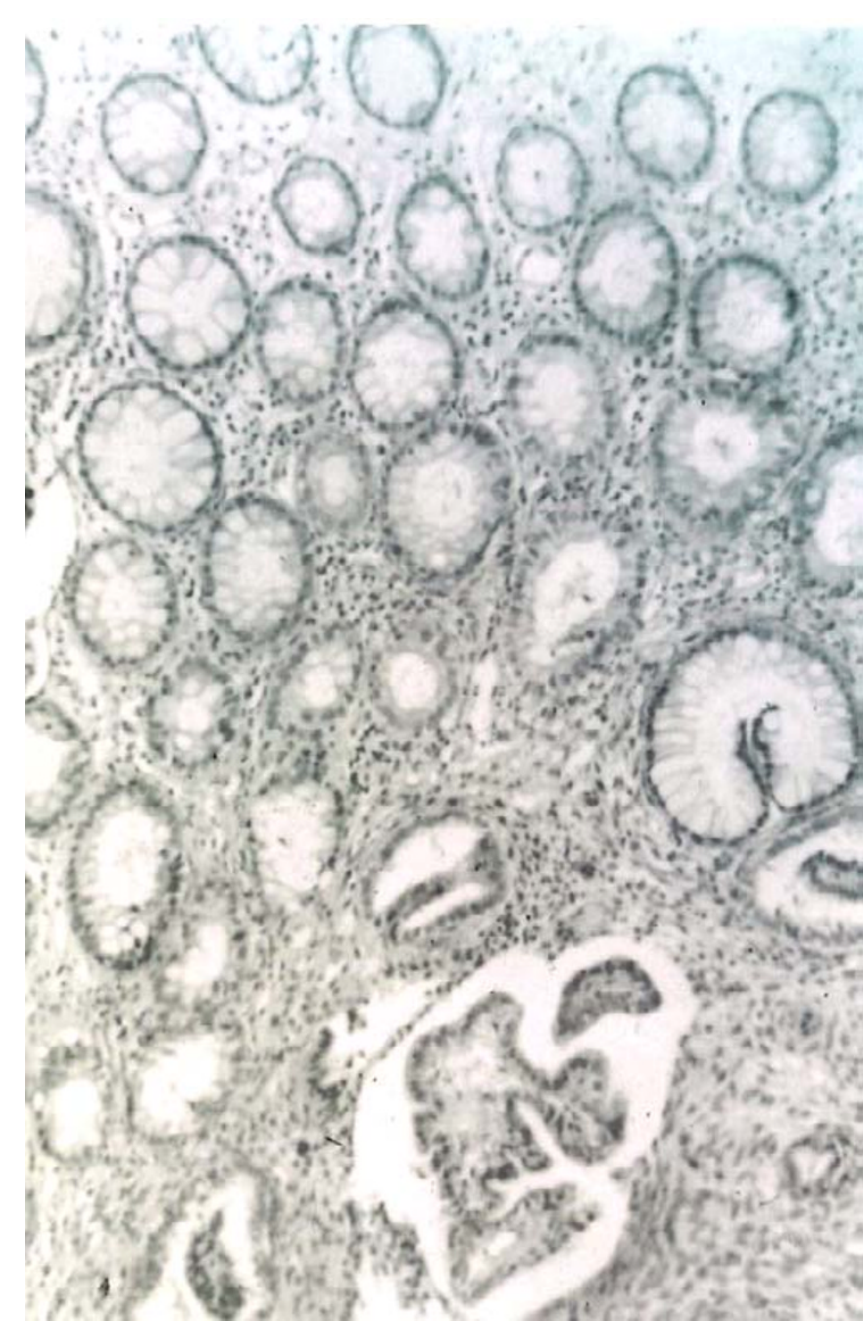

Figure 4

Large bowel mucosa with a focus of endometriosis in the lamina propria (stroma and glands of endometrium). Haematoxylin Eosin $\times 100$

Five months later a new colonoscopy was performed that showed only mild oedema of the sigmoid mucosa with disappearance of the stenotic lesion and easy introduction of the instrument to the caecum. The rest of the large bowel appeared to be normal. Serum level of CA-125 (7th day of menstrual cycle) was now normal $(28 \mathrm{IU} / \mathrm{ml})$. The patient was free of symptoms.

\section{Discussion}

The gastrointestinal tract is the most common site of extrapelvic endometriosis, affecting $5-15 \%$ of women with pelvic endometriosis [1,2]. Among women with intestinal endometriosis, rectum and sigmoid colon are the most common involved areas (75-90\%). Other parts 
of the bowel commonly affected are the distal ileum (2$16 \%)$, and appendix (3-18\%) [3]. Only the serosa and the muscularis propria are usually involved, while the mucosa is very rarely affected [4].

Intestinal endometriosis may present with rectal bleeding, bowel obstruction and rarely with perforation or malignant transformation $[5,6]$. Symptoms can be cyclical in about $40 \%$ of patients, can vary depending on the site and include crampy abdominal pain, distention, diarrhea, constipation, tenesmus and hematochezia [1]. The classic triad of dysmenorrhea, dyspareunia and infertility, as a result of concomitant pelvic disease, may also exist. We should, however notice, that the cyclical character of symptoms does not exclusively appear in endometriosis. It is well established that clinical manifestations in inflammatory bowel disease and irritable bowel syndrome may aggravate during the menses $[7,8]$.

The clinical, radiological and endoscopic picture may be confused with neoplasms, ischemic colitis, inflammatory bowel disease, post radiation colitis, diverticular disease and infection. Although endoscopic diagnosis of colonic endometriosis has been reported [9] usually the endoscopic appearance, even if there is mucosal involvement, is not diagnostic. Biopsies obtained endoscopically usually yield insufficient tissue for a definite pathologic diagnosis [10]. Moreover, endometriotic deposits can induce secondary mucosal changes, which mimic findings of other diseases such as inflammatory bowel disease, ischemic colitis, or even a neoplasm $[11,12]$. The CT scan or barium enema usually demonstrate an extrinsic bowel compression, stenosis or filling defect. MRI seems to be the most sensitive imaging technique for intestinal endometriosis [13]. Yet, the gold standard for the diagnosis is laparoscopy or laparotomy.

Treatment options include surgery or hormonal manipulations, depending on patient's age and desire to maintain fertility and also on the severity and complications of the disease [14]. Recently, laparoscopic treatment of colorectal endometriosis, even in advanced stages, has been proven feasible and effective in nearly all patients [15]. The medications used in the treatment of endometriosis are danazol, high dose progestins and GnRH agonists with almost equivalent efficacy [16]. The choice of which to use is based on side effects and costs. Danazol and GnRH agonists are of equivalent cost, but GnRH agonists are usually better tolerated. In our case, although high dose progestins could have been used, after discussion with the patient she chose to receive GnRH agonists.

Our patient represents a case of symptomatic gastrointestinal endometriosis with mucosal involvement, without a previous history of pelvic endometriosis. The symptoms of abdominal pain, constipation and hematochezia, the presence of anemia in combination with the radiologic and endoscopic findings were suggestive of a neoplasm. On the other hand the patient's long history of dysmenorrhea, the normal levels of CEA and CA19-9, and the absence of neoplastic infiltration in all biopsy specimens were against the diagnosis of colon cancer. Furthermore, the ovarian chocolate cysts showed by the pelvic ultrasound and the histologic evidence, obtained from the second colonoscopy, directed us towards the diagnosis of intestinal endometriosis that was finally confirmed during the laparoscopy. Moreover, this patient had elevated serum levels of CA-125, which has been established as a useful marker for determining the severity of endometriosis $[17,18]$. The colonic mucosal involvement in this case could be explained by the invasion of endometrial cells through the bowel wall. Lymphatic or vascular metastasis could explain rare cases of endometriosis located in pleura, umbilicus, muscle, brain, vagina, cervix and retroperitoneal space $[1,2]$.

In conclusion, intestinal endometriosis is often a diagnostic challenge mimicking a broad spectrum of diseases and should be considered in any young woman with symptoms from the lower gastrointestinal tract.

\section{List of abbrevations}

CEA, carcinoembryonic antigen; CNS, central neural system; CT, computer tomography; GnRH, gonadotropinreleasing hormone; MCV, mean corpuscular volume; MRI, magnetic resonance imaging;

\section{Competing interests}

None declared.

\section{Acknowledgements}

Written consent was obtained from the patient for publication of the patient's details.

\section{References}

I. Jubanyik K and Comite F: Extrapelvic endometriosis. Obstet Gynecol Clin North Am 1997, 24:4 I I-40.

2. Schwartz JL and Schwartz LB: Extrapelvic endometriosis. In Endometrium and Endometriosis Edited by: Diamond MP, Osteen KG. Blackwell Science; 1997:247-254.

3. Miller LS, Barbarevech C and Friedman LS: Less frequent causes of lower gastrointestinal bleeding. Gastroenterol Clin North Am 1994, 23:21-52.

4. Levitt MD, Hodby KJ, van Merwyk AJ and Glancy RJ: Cyclical rectal bleeding in colorectal endometriosis. Aust N Z J Surg 1989 , 59:941-3.

5. Varras M, Kostopanagiotou E, Katis K, Farantos Ch, Angelidou-Manika $\mathrm{Z}$ and Antoniou S: Endometriosis causing extensive intestinal obstruction simulating carcinoma of the sigmoid colon: a case report and review of the literature. Eur J Gynaecol Oncol 2002, 23:353-7.

6. Yantiss RK, Clement PB and Young RH: Neoplastic and pre-neoplastic changes in gastrointestinal endometriosis: a study of I 7 cases. Am J Surg Pathol 2000, 24:5I3-24.

7. Kane SV, Sable K and Hanauer SB: The menstrual cycle and its effect on inflammatory bowel disease and irritable bowel 
syndrome: a prevalence study. Am J Gastroenterol 1998, 93:1867-1872

8. Heitkemper MM, Cain KC, Jarrett ME, Burr RL, Hertig V and Bond EF: Symptoms across the menstrual cycle in women with irritable bowel syndrome. Am J Gastroenterol 2003, 98:420-30.

9. Bozdech JM: Endoscopic diagnosis of colonic endometriosis. Gastrointest Endosc 1992, 38:568-70.

10. Scully RE, Mark EJ, McNeely WF, Ebeling SH and Ellender SM: Case Records of the Massachusetts General Hospital (Case 132000). N Engl J Med 2000, 342: I 272-1 278.

II. Barcclay RL, Simon JB, Vanner SJ, Hurlbut DJ and Jeffrey JF: Rectal passage of intestinal endometriosis. Dig Dis Sci 200I, 46:1963-1967.

12. Langlois NEI, Park KGM and Keenan RA: Mucosal changes in the large bowel with endometriosis: a possible cause of misdiagnosis of colitis? Hum Pathol 1994, 25: 1030-4.

13. Brosens J, Timmerman D, Starzinski-Powitz A and Brosens I: Noninvasive diagnosis of endometriosis: the role of imaging and markers. Obstet Gynecol Clin North Am 2003, 30:95-I I4.

14. Urbach DR, Reedijk M, Richard CS, Lie KI and Ross TM: Bowel resection for intestinal endometmetriosis. Dis Colon Rectum 1998, 41: I | 58-64.

15. Jerby BL, Kessler H, Falcone T and Milsom JW: Laparoscopic management of colorectal endometriosis. Surg Endosc 1999, 13:1125-8.

16. Mahutte NG and Arici A: Medical Management of Endometriosis-Associated Pain. Obstet Gynecol Clin North Am 2003, 30: I33-50.

17. Harada $T$, Kubota $T$ and Aso $T$ : Usefulness of CAI 9-9 versus CAI25 for the diagnosis of endometriosis. Fertil Steril 2002, 78:733-9.

18. Matalliotakis I, Makrigiannakis A, Karkavitsas N, Psaroudakis E, Froudarakis $G$ and Koumantakis $E$ : Use of CA-I 25 in the diagnosis and management of endometriosis: influence of treatment with danazol. Int J Fertil Menopausal Stud 1994, 39:100-4.

\section{Pre-publication history}

The pre-publication history for this paper can be accessed here:

http://www.biomedcentral.com/1471-230X/3/18/pre

pub

Publish with Bio Med Central and every scientist can read your work free of charge

"BioMed Central will be the most significant development for disseminating the results of biomedical research in our lifetime. "

Sir Paul Nurse, Cancer Research UK

Your research papers will be:

- available free of charge to the entire biomedical community

- peer reviewed and published immediately upon acceptance

- cited in PubMed and archived on PubMed Central

- yours - you keep the copyright 